\title{
L'inscription paysagère du risque d'inondation dans les politiques urbaines des agglomérations ligériennes, proposition d'un marqueur de résilience spatiale
}

The landscaped retranscription of the risk of flooding in the urban policies of the agglomerations of the Loire Valley: proposition of a marker of spatial resilience

Bertrand Sajaloli, Sylvie Servain-Courant, Sylvain Dournel et Dominique Andrieu

\section{OpenEdition}

\section{Journals}

Édition électronique

URL : http://journals.openedition.org/rge/3439

DOI : $10.4000 /$ rge.3439

ISSN : 2108-6478

Éditeur

Association des géographes de l'Est

Édition imprimée

Date de publication : 23 août 2011

ISSN : 0035-3213

Référence électronique

Bertrand Sajaloli, Sylvie Servain-Courant, Sylvain Dournel et Dominique Andrieu, «L'inscription paysagère du risque d'inondation dans les politiques urbaines des agglomérations ligériennes, proposition d'un marqueur de résilience spatiale », Revue Géographique de l'Est [En ligne], vol. 51 / 3-4 | 2011, mis en ligne le 28 août 2012, consulté le 08 septembre 2020. URL : http:// journals.openedition.org/rge/3439; DOI : https://doi.org/10.4000/rge.3439

Ce document a été généré automatiquement le 8 septembre 2020

Tous droits réservés 


\section{L'inscription paysagère du risque d'inondation dans les politiques urbaines des agglomérations ligériennes, proposition d'un marqueur de résilience spatiale}

The landscaped retranscription of the risk of flooding in the urban policies of the agglomerations of the Loire Valley: proposition of a marker of spatial resilience

Bertrand Sajaloli, Sylvie Servain-Courant, Sylvain Dournel et Dominique Andrieu

\section{Introduction}

L'intégration du risque d'inondation dans les politiques urbaines durables ${ }^{1}$ modifie le regard des collectivités locales et des urbanistes sur les zones inondables, bâties ou non bâties ${ }^{2}$. Hier encore négligées, voire conspuées et supprimées ${ }^{3}$, ces zones humides guident aujourd'hui bon nombre de projets urbains ${ }^{4}$ et renforcent voire reconstruisent l'identité de nombreuses cités fluviales ${ }^{5}$. Ces lieux d'eau revêtent en effet de nouveaux enjeux d'aménagement (nature en ville, rôle d'infrastructure naturelle, mixité sociale) et supportent des fonctions inédites (trames bleues, trames vertes) afin notamment d'atténuer la vulnérabilité urbaine au risque hydrologique et d'en accroître la résilience. Mais si ces deux enjeux nourrissent le projet de ville durable, ils peinent à trouver un contenu paysager et à s'inscrire spatialement dans les documents d'urbanisme. Ceci motive cette étude qui, par une démarche en trois temps, s'appuie sur les trois principales agglomérations de la Loire moyenne (Orléans, Blois et Tours) depuis l'instauration des Plans de prévention des risques d'inondation (PPRI) au début des années 2000. 
2 Dans un premier temps, à partir des documents cartographiques ou iconographiques disponibles et d'enquêtes de terrain, les aménagements urbains récents (parcs urbains, espaces verts) ou plus anciens (zones agricoles, zones d'activités) situés en zones inondables sont caractérisés dans les secteurs marqués par un fort aléa (niveaux 3 et 4 $\mathrm{du}$ PPRI). Il s'agit de voir en quoi les politiques urbaines intégrant le risque d'inondation façonnent de nouveaux paysages et induisent de nouvelles pratiques de la ville. Cette analyse débouche sur une typologie des espaces créés, recomposés ou ignorés par l'intégration du risque dans les politiques urbaines, depuis le lancement des PPRI. Dans un deuxième temps, une grille paysagère d'évaluation de la résilience urbaine au risque d'inondation, ou plus exactement de la capacité à faire face au risque (coping capacity) ${ }^{6}$, est confectionnée grâce à des marqueurs révélant la vulnérabilité ou au contraire l'adaptation de l'unité paysagère à l'aléa encouru. Chaque marqueur est doté d'une appréciation chiffrée permettant les comparaisons inter-sites et attribuant à chaque catégorie de paysage un indice de résilience, ou plus modestement de réaction au risque. D'abord testée sur le val inondable d'Orléans, cette méthode est ensuite appliquée sur les trois sites. Les synthèses spatiales sont enfin réalisées grâce à l'utilisation d'un Système d'Informations Géographiques homogènes commun à chacune des agglomérations ligériennes étudiées : il en ressort une appréciation à la fois spatialisée et hiérarchisée de la capacité à faire face au risque d'inondation. Enfin, en croisant les deux temps précédents, il s'agit d'estimer en quoi l'intégration du risque d'inondation concourt à la fabrique de villes résilientes.

3 La notion de résilience spatiale est donc au centre de la réflexion, notamment dans son contenu paysager qui offre des possibilités inédites d'intégrer la capacité à faire face au risque à l'échelle des documents d'urbanisme. Pourtant, cette notion n'est ici qu'approchée. D'une part, la résilience ne peut stricto sensu être étudiée que s'il y a eu une catastrophe au préalable. Nous l'avons néanmoins retenue pour ses effets prédictifs et afin de voir en quoi, davantage que la vulnérabilité, elle aide à mettre en place une politique de prévention des risques. En effet, puisque le risque d'inondation est dominant dans les trois sites étudiés, puisque la catastrophe ancienne est connue $(1846,1856,1866)$, projeter l'aléa d'hier sur le terrain d'aujourd'hui laisse envisager la résistance des unités spatiales et leur coping capacity. On s'approche ainsi de la notion de résilience. D'autre part, la résilience intègre les inter-relations et les interdépendances entre les espaces et accorde à leur connectivité une importance cruciale. À cet égard, notre approche n'est que préparatoire et débute par une réflexion posant les cadres conceptuels de la réflexion.

\section{Résilience spatiale et systèmes urbains : vers une grille d'évaluation paysagère de la résilience urbaine en Val de Loire}

\section{A. L'impasse paysagère de la notion de résilience urbaine}

4 La notion de résilience, avancée pour l'écologie ${ }^{7}$ et peu à peu appliquée à des systèmes spatiaux anthropisés ${ }^{8}$, tend à acquérir une définition de plus en plus stable ${ }^{9}$ et à déterminer des politiques territoriales ou à créer des outils d'aide à la décision, notamment à l'issue ou à l'approche de crises environnementales ${ }^{10}$. Confrontée à l'espace urbain, elle est à l'origine du concept de ville résiliente ${ }^{11}$, lui aussi forgé en 
regard d'un événement environnemental catastrophique ayant pu, ou devant, bouleverser villes et métropoles ${ }^{12}$. Si les grandes cités nord-américaines bénéficient aujourd'hui bien plus souvent du qualificatif de ville résiliente (Détroit, Montréal, Québec, Vancouver...), cet adjectif, tend également à s'accoler aux grandes agglomérations exposées à des risques naturels majeurs (Tokyo) ou ayant subi des traumatismes géopolitiques importants (Beyrouth). Enfin, ce n'est que très récemment qu'est explorée en France la force opérationnelle du concept de ville résiliente ${ }^{13}$, notamment grâce au programme PIRVE ${ }^{14}$.

5 Cette émergence conceptuelle correspond à un glissement assez général de la notion de vulnérabilité ${ }^{15}$ à celle de résilience, sans toutefois que les présupposés systémiques soient toujours clairement exposés. Il est en effet frappant que les travaux de C. Aschan ${ }^{16}$ soient si peu repris et que la résilience soit, et c'est souvent la vision dominante au sein des collectivités territoriales, le plus souvent assimilée à la notion d'un retour à la stabilité d'un système autour d'un point d'équilibre. Sur un plan géographique, un espace résilient serait alors, rêve spatial de beaucoup d'élus et de citadins, celui qui verrait resurgir le même mode d'occupation du sol qui le caractérisait avant l'événement catastrophique. Ceci pose à l'évidence la question de la résilience spatiale qui implique au contraire que le géosystème urbain maintienne ses structures qualitatives et assure sa continuité, non pas en figeant un équilibre ancestral mais en intégrant des transformations susceptibles de le faire évoluer positivement ${ }^{17}$. Dans ce contexte de systèmes ouverts, qui supposent tout à la fois l'existence de plusieurs situations d'équilibre et le fait qu'un système peut perdurer loin d'un équilibre sans autant disparaître, la dimension historique apparaît primordiale. En effet, la géohistoire du système urbain ${ }^{18}$ permet tout à la fois de repérer les crises et mutations de l'espace, mais aussi la capacité adaptative du système considéré et quelques unes de ses situations d'équilibres fluctuants. Ceci évite la réitération d'un certain nombre d'erreurs d'aménagement, et autorise aussi, en intégrant les facteurs d'identité socio-spatiaux, l'édiction de scenarii d'évolutions susceptibles de guider la décision politique. Mais pour être concrets, et pris en compte par les collectivités territoriales, ces scenarii évolutifs ne doivent pas seulement reposer sur des modèles de transition, comme celui de cycle adaptatif proposé par le groupe Résilience Alliance ${ }^{19}$ qui distingue quatre phase ${ }^{20}$ et débouche sur le principe de gestion adaptative ${ }^{21}$. En effet, la dimension spatiale, considérée à l'échelle du paysage ${ }^{22}$, est primordiale afin que la notion de résilience puisse s'inscrire effectivement dans les politiques publiques. Dès lors, notre objectif est de proposer, à l'échelle des documents d'urbanisme (Plan local d'urbanisme, Plan d'occupation des sols ayant valeur de PLU), une grille d'évaluation de la résilience urbaine.

\section{B. Le système spatial des cités ligériennes}

6 Les cités ligériennes se prêtent remarquablement à cet exercice. D'une part, elles font l'objet de programmes de recherches ${ }^{23}$ qui ont permis une bonne connaissance de la vulnérabilité du système ligérien ${ }^{24}$ et des modalités politiques et sociales de la prise en compte du risque d'inondation ${ }^{25}$. D'autre part, elles peuvent être perçues comme des systèmes en mutation, voire en crise, l'urbanisation massive du val inondable étant particulièrement récente et se poursuivant malgré les préconisations du PPRI. À cet égard, nous ne retiendrons qu'un seul exemple : le val d'Orléans accueille aujourd'hui plus de 48000 personnes dans une zone inondable caractérisée par des aléas forts 
(niveaux 2, 3 et 4) et qui seraient prises au piège des eaux en cas d'une crue de retour 200 ans $^{26}$. Enfin, la conscience politique du risque, aiguillonnée par des injonctions réglementaires nouvelles (révision du PPRI, notamment), conduit les édiles urbains à anticiper les effets du risque sur leurs territoires communaux ${ }^{27}$. L'enjeu scientifique est donc double. Il s'agit d'adopter une lecture systémique de l'espace urbain inondable et de poser la question de la résilience au niveau de la grande échelle des documents d'urbanisme.

$7 \quad$ La lecture systémique de l'espace urbain inondable repose sur l'étude approfondie des modes historiques de valorisation $\mathrm{du} \mathrm{sol}^{28}$. Il en ressort, d'une part, que les zones les plus exposées (aléas 3 et 4 du PPRI) ont sans cesse fait l'objet de mises en valeur intensives (économies pastorales jusqu'au milieu du XIXe, viticole jusqu'à la Première Guerre mondiale, maraîchère et intensive jusqu'aux années 1960). D'autre part, l'ampleur du risque, notamment lors des épisodes catastrophiques de 1846, 1856 et 1866, avait d'autant plus préservé de l'urbanisation ces secteurs, pourtant situés au cœur des agglomérations ligériennes, que les productions choisies étaient fortement rémunératrices. Par-delà la diversité des sociétés rurales et urbaines considérées, il y avait bien là un système spatial ligérien qui, évolutif, tendait à répartir les activités, et ainsi les paysages, selon des gradients d'exposition au risque et de vulnérabilité. Dès lors, l'urbanisation récente, principalement composée de pavillons de plain-pied qui seraient tout ou partie immergés par les eaux ${ }^{29}$, peut bien être perçue comme une crise $\mathrm{du}$ système spatial. Affirmer ceci n'induit en rien que nous considérions l'urbanisation comme un méfait et que nous prêtions aux seuls espaces naturels et agricoles des vertus résilientes. Au contraire, dans le passé, des habitats sur tertres, levées ou terrasses surélevées ont fixé les noyaux des premières cités en les maintenant hors d'eau. De même, l'histoire des aménagements hydrauliques de la Loire destinés à contenir les crues a fortement modifié la géographie du risque. Enfin, de nouvelles techniques architecturales permettraient la construction d'habitation en zones fortement inondables; c'est d'ailleurs ce que préconise la ville d'Orléans en ayant adhéré au réseau européen Flood Resilient Cities ${ }^{30}$.

8 La question de l'échelle dans la caractérisation de la résilience pose un paradoxe géographique bien connu ${ }^{31}:$ la détermination et l'anticipation des systèmes spatiaux sont d'autant plus aisées que l'espace est étendu. À l'inverse, la grande échelle géographique (la commune, le quartier, la parcelle) induit souvent une forme de "bruit " ${ }^{32}$, de perturbation territoriale, venant gêner la définition ou l'application du modèle systémique. Il est d'ailleurs remarquable que certains auteurs aient relevé ces difficultés scalaires mentionnant l'impossible territorialisation du risque en ville ${ }^{33}$ ou au contraire les liens entre savoir cartographique et pouvoir bureaucratique ${ }^{34}$. De même, les trois sites étudiés de Saint-Pryvé-Saint-Mesmin, Blois rive-droite et La Riche constituent autant de systèmes spatiaux élémentaires s'insérant dans des systèmes propres à chacune des agglomérations (Orléans, Bois et Tours) et, au plus petit niveau scalaire, dans le système global des cités ligériennes. C'est pourquoi, à notre connaissance, les tentatives d'établir des cartes de résilience spatiale à grande échelle susceptibles de constituer des outils d'aide à la décision sont peu nombreuses. 


\section{Vers une grille d'évaluation paysagère de la résilience urbaine}

9 La méthode expérimentale proposée repose sur les paysages pour spatialiser la résilience urbaine à grande échelle. S'appuyant sur des études ligériennes ${ }^{35}$ et rhodaniennes ${ }^{36}$, elle a été élaborée dans le Val d'Orléans ${ }^{37}$ à partir des documents cartographiques et d'urbanisme, des orthophotoplans récents et missions aériennes anciennes et de minutieuses enquêtes de terrain ${ }^{38}$. L'analyse paysagère est fondée sur la présence ou l'absence de caractéristiques qui visent à évaluer la résilience de l'espace. Davantage que sur des conceptions holistiques du paysage, la méthode s'appuie donc tout à la fois sur des perspectives géosystémiques, où l'unité paysagère composée par chaque mode spécifique d'occupation du sol constitue le plus petit élément du système spatial, et sur l'utilisation de marqueurs et de signes paysagers attestant des formes et intensité de la résilience. Quatre critères principaux ont été retenus : l'occupation du sol, les fonctions hydrologiques, la vulnérabilité et la pédagogie. Le premier s'appuie sur une typologie des modes d'occupation du sol qui reprend les grandes logiques des Plans locaux d'urbanisme afin d'établir des liens entre cartographie de la résilience et documents de gestion. Le deuxième, à partir des types présentiels des espaces en eau ou susceptibles de stocker ou d'évacuer l'eau, rend compte des fonctions hydrauliques et hydrologiques en cas de crue : écouler, stocker, ralentir et freiner l'écoulement des eaux, alimenter les nappes. Chaque item a été doté d'une note, de 0 à 2 , suivant les capacités de l'élément paysager considéré à évacuer ou à supporter l'eau. Au total, le critère hydrologique a été noté sur 8 . Le troisième, noté sur 7 , reprend les études sur la vulnérabilité du bâti et des espaces non bâtis et introduit la notion de pérennité des paysages. En effet, certains aménagements ou modes d'occupation du sol conçus pour avoir une durée de vie réduite peuvent être submergés sans perdre leurs potentialités. De tels espaces sont dotés d'une note de deux contrairement à des aménagements plus lourds et moins adaptés à un risque de crue qui bouleverseraient la valorisation post aléa de l'espace. Ceux-ci obtiennent une note de zéro. L'intérêt et la valorisation du patrimoine naturel tiennent une place importante dans des espaces soumis aux inondations du fleuve car ils permettent le maintien d'un bon fonctionnement hydrologique du système fluvial. Enfin, le dernier critère, noté sur 5 , s'appuie sur les marques paysagères de la communication du risque au public fréquentant ou habitant à proximité. Cette pédagogie du risque s'inscrit en droit-fil des préconisations réglementaires en matière de sensibilisation des populations (Plan communal de sauvegarde, Document d'information communal sur les risques majeurs) et reprend les études conduites sur la perception du risque par les habitants ${ }^{39}$. À cet égard, l'ouverture du paysage sur l'espace ligérien est considérée comme un marqueur de communication et de considération du risque dans la ville. Le paysage présente trois degrés d'ouverture à la Loire : aucune vue sur la Loire ( 0 point), vue partielle sur la Loire (1 point), vue globale sur la Loire (2 points). Le tableau 1 reprend les temps forts de la méthodologie proposée : chaque unité paysagère élémentaire se voit dotée d'une note de 0 à 20 selon qu'elle n'est pas du tout (0) ou très (20) résiliente. Testée à Orléans, cette méthode expérimentale a ensuite été adaptée et appliquée aux deux autres cités ligériennes afin de disposer d'une approche commune. 
Tableau 1 : Grille paysagère d'évaluation de la résilience urbaine au risque d'inondation

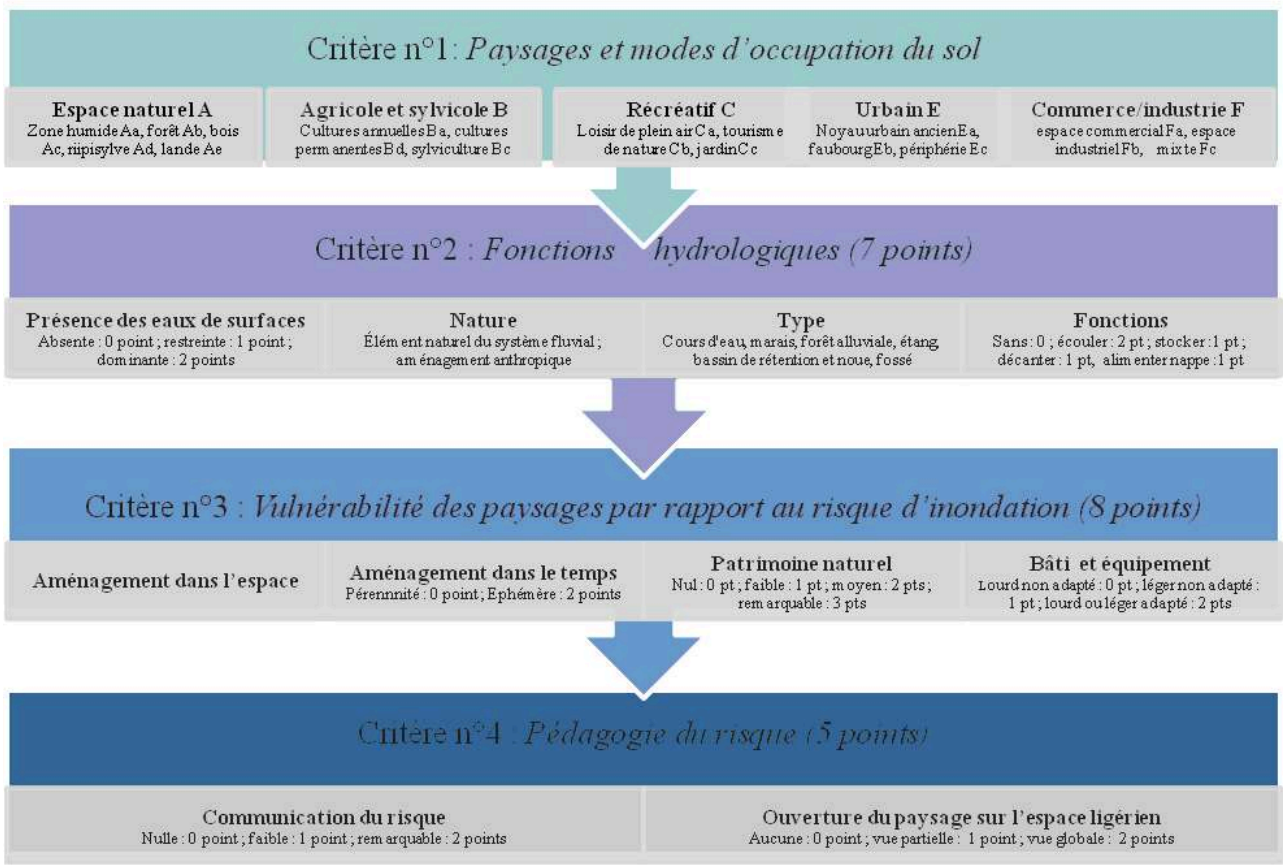

La fréquentation assidue du terrain et l'existence de nombreux tests, notamment destinés à évaluer le poids relatif de chaque critère dans la notation finale, ont émaillé cette approche qui reste pourtant essentiellement qualitative. Elle n'en constitue pas moins à nos yeux une première tentative de cartographier la résilience spatiale urbaine à grande échelle.

\section{Applications à trois villes ligériennes vulnérables : Saint-Pryvé-Saint-Mesmin (Orléans, Loiret), Blois (Loir-et-Cher) et La Riche (Tours, Indre-et-Loire)}

\section{A. Orléans, Blois, Tours : des villes à l'épreuve du risque}

11 Les agglomérations d'Orléans, Blois et de Tours, et plus particulièrement, la commune de Saint-Pryvé-Saint-Mesmin, le quartier Blois rive-gauche et la commune de La Riche, constituent des sites d'investigation propices à une réflexion sur la résilience spatiale en raison du risque d'inondation de la Loire et de ses affluents, de l'urbanisation récente des zones les plus exposées au risque et de la pression foncière qui s'y poursuit. Considérée comme l'une des catastrophes naturelles prévisibles les plus importantes en France métropolitaine, une crue majeure (www.r.inondation-loire.fr et www.prim.net ${ }^{40}$ ) y provoquerait de lourds dommages économiques ${ }^{41}$. En outre, l'hydrosystème ligérien en place est atypique: non seulement, il témoigne d'un façonnement anthropique pluriséculaire ${ }^{42}$, mais encore il conserve un fort caractère de naturalité. Le val de Loire, ponctué de châteaux, d'édifices religieux et de fronts urbains à caractère patrimonial, est inscrit depuis 2002 sur la liste du patrimoine mondial de l'UNESCO au titre des paysages culturels évolutifs. 

terminaison d'un vaste val inondable, étendu entre Loire et Loiret et entre centre historique et ville nouvelle de La Source. En 2007, sur un total de 275442 habitants au sein de la communauté d'agglomération, 48000 personnes vivent en zone inondable, soit $18 \%$ du total. Sur un plan économique, cela représente 1430 entreprises et $12 \%$ des emplois. La commune de Saint-Pryvé-Saint-Mesmin (5 408 habitants en 2007) fait office de commune-test pour notre étude. Ce bourg du sud-ouest de l'agglomération orléanaise a en effet enregistré une forte croissance urbaine entre 1954 et 1990, passant de 1537 à 5463 habitants, alors qu'il est intégralement exposé au risque d'inondation, avec des plus hautes eaux connues à plus de trois mètres sur la majeure partie de son territoire (crues de 1846, 1856 et 1866). Sa proximité du centre d'Orléans $(4 \mathrm{~km})$, ses grandes disponibilités foncières liées à une agriculture en difficulté et son cadre de vie de qualité entre Loire et Loiret, expliquent cette dynamique ${ }^{43}$.

13

Plus en aval, la ville de Blois, chef-lieu du département du Loir-et-Cher, au cœur du Val de Loire, est également soumise aux problématiques des constructions en zone inondables. Inondée à trois reprises au XIXe siècle, plus précisément en 1846, 1856, et 1866 , elle a connu également des submersions, certes moins significatives, en 1907, qui ont pu être limitées grâce à l'existence d'un déversoir ayant détourné une partie du débit de crue. Enfin, si en 2003 la côte d'alerte a été dépassée, le fleuve est resté dans son lit. La Loire coupe donc la ville en deux zones : le plateau de la rive nord, hors d'eau qui concentre la majorité de la population, des équipements et des pôles d'emplois, la rive sud principalement concernée par le risque d'inondation. Deux quartiers, Vienne et la Bouillie, situés entre la Loire et le Cosson, concentrent aujourd'hui les attentions locales en matière de prise en compte des inondations dans l'aménagement. Le premier en aléa 2 du PPRI (fig. 3) est protégé par une digue, le second, en aléa 4, fait l'objet d'une opération très originale de désurbanisation ${ }^{44}$.

Troisième terrain d'étude, l'agglomération de Tours, localisée plus en aval, est dans un site de confluence, entre Loire et Cher. Le cœur de l'agglomération est situé en zone inondable puisque s'y trouvent le centre historique de Tours ainsi que des zones regroupant des activités économiques importantes, que cela soit le centre commercial des Atlantes, le Parc des expositions ou la gare ferroviaire de Saint-Pierre-des-Corps. En 2006, 22\% des surfaces de l'agglomération sont situées en zone inondable, regroupant $36 \%$ d'une population de près de 307000 habitants. La commune de La Riche se situe à l'ouest de Tours, dans la continuité du tissu urbain dense et proche du centre-ville. Longtemps enclavé, car limitée au nord par la Loire et au sud par le Cher, le territoire n'a été plus facilement accessible qu'après la construction du périphérique ouest et des ponts l'accompagnant, dans les années 1990. Son attractivité s'est alors renforcée : de 1990 à 2010, la population est passée de 7800 à plus de 10000 habitants. Cette croissance s'accompagne d'une transformation du territoire et de ses paysages. Grands ensembles, lotissements de maisons individuelles, implantation de zones industrielles, favorisées par la présence de deux lignes de chemin de fer, prennent la place des terres agricoles, dont des parcelles de maraîchage et de jardins. Le développement urbain suit les routes; les principales étant orientées parallèlement aux cours d'eau et progresse d'est en ouest. Un visage périurbain prend peu à peu forme, reléguant les terres agricoles en marge de la ville. 


\section{B. Le risque, un moteur paysager}

15 À partir de la méthode expérimentée à Orléans ${ }^{45}$, les effets paysagers et fonctionnels de l'intégration (ou du déni) du risque dans les paysages urbains situés en zone inondable ont fait l'objet d'une typologie en trois catégories appliquées aux trois sites. Elle distingue des espaces créés, recomposés ou ignorés par la prise en considération du risque dans les politiques urbaines depuis le lancement des PPRI et se déclinent en 13 sous classes (tableau 2). On voit que l'intégration du risque constitue un puissant moteur de nouveaux paysages et usages : espaces récréatifs et sportifs (I.1) jouxtent des systèmes hydrauliques de contrôle des eaux pluviales formant de grands bassins aménagés (I.3) tandis que la mise aux normes de stations d'épuration prévoit leur submersion (I.4). De même, la protection de la nature en milieu intra-urbain (I.5) est largement soutenue par la vulnérabilité des lieux en cas de crue ; plus original encore, l'espace agricole peut être classé en zone $\mathrm{N}$ au sein des PLU afin de le soustraire à toute urbanisation. Ainsi, avec les objectifs de nature en ville, de mixité sociale, avec les créations de trames vertes et bleues constituant des infrastructures naturelles, assistet-on à la fabrique de la ville durable. La deuxième sous-classe concerne les aménagements et paysages maintenus mais assortis de nouvelles fonctionnalités et réglementations adaptées au risque d'inondation. On y trouve l'ensemble des espaces agricoles, directement hérités des valorisations traditionnelles des zones submersibles, mais maintenant assortis d'une interdiction d'urbanisation (II.1), les zones naturelles ne bénéficiant pas d'un statut de protection mais classées en zone N (II.2), les voieries et infrastructures de transport hors d'eau (II.3) et enfin les aires d'extraction de granulat dont l'aménagement et la reconversion tiennent compte du risque d'inondation (II.4). L'accent est mis ici sur les héritages historiques de la valorisation agricole des espaces périurbains ainsi que sur la reconnaissance de la nature banale. Subsistent toutefois des espaces anciens, et parfois même récents, qui, n'intégrant nullement le risque d'inondation, présentent forte vulnérabilité et faible résilience. Ils rassemblent les zones bâties en zone inondable avant le PPRI (III.1), les espaces en cours d'aménagement (III.2) qui profitent du vide juridique lié à la prochaine révision des PPRI ${ }^{46}$, les zones naturelles, souvent en marge des territoires communaux qui sont marquées par une occupation illégale de l'espace (habitat précaire, notamment associé aux gens du voyage en cours de sédentarisation) (III.3) et enfin les secteurs liés aux activités industrielles ou commerciales dont l'activité se poursuit malgré l'exposition aux inondations (III.4). Cette typologie tripartite, appliquée sur les trois agglomérations étudiées, testée sur les agglomérations ligériennes de Nevers à Angers, regroupe ainsi la majeure partie des cas rencontrés. Il y manque pourtant, notamment à Angers, les rares mais exemplaires cas de construction du XXI siècle sur dalles rehaussées ou pilotis et également, l'ensemble des bâtiments anciens qui concentraient activités et habitats sur les étages supérieurs de hautes bâtisses. 
Tableau 2 : Intégration du risque et création de paysages résilients

\begin{tabular}{|c|c|c|}
\hline Catégorie & Sous-catégories & $\begin{array}{l}\text { Evaluation } \\
\text { résilience }\end{array}$ \\
\hline \multirow{5}{*}{$\begin{array}{l}\text { I - Noureaux espaces } \\
\text { créés par } \\
\text { l'integration du } \\
\text { risque d'inondation } \\
\text { dans les dyamiques } \\
\text { spatiales urbaines }\end{array}$} & $\begin{array}{l}\text { (1) espaces récréatifs et sportifs susceptibles d'être } \\
\text { tubmergés sans provoguer de dégâts matériels importants }\end{array}$ & 12 a 16 \\
\hline & $\begin{array}{l}\text { 2) zones agricoles récemment classées en zone } \mathrm{N} \text { afin de lef } \\
\text { eler a toute urbanisation }\end{array}$ & 13 à 18 \\
\hline & $\begin{array}{l}\text { 3) systèmes hydrauliques de contrôle des eaux pluvialeg } \\
\text { bassin de rétention, noues drainantes, fossés) pouvan } \\
\text { vventuellement être untlisées à des fins récréatives }\end{array}$ & 13 à 18 \\
\hline & 4) stations d'épuration ayant integré le nsoue d'mondation & 14 an1 \\
\hline & $\begin{array}{l}\text { (5) espaces naturels bénéficiant d'un statut de protectiog } \\
\text { églementaive (Arrété de biotope, zone Natura } 2000 \text {, réserve } \\
\text { aturelle) afin d'en maintenir la biodiversité et de respecter } \\
\text { es cycles hydrologioues majeurs de la Loire }\end{array}$ & $16 \mathrm{a} 20$ \\
\hline \multirow{4}{*}{$\begin{array}{l}\text { II - Espaces } \\
\text { maintenus mais } \\
\text { assortis de nouvelles } \\
\text { réglementations et } \\
\text { fonctionnalités } \\
\text { intégrant le risque } \\
\text { d'inondation }\end{array}$} & $\begin{array}{l}\text { (1) différentes catégonies d'espace agnicole (vigne, vergers } \\
\text { épinières, maraíchage, prairie exploitée ou non) classées en } \\
\text { one } \mathrm{A} \text { et assorties d'une interdiction d'ubanisation en } \\
\text { lehors des secteurs de faible aléa }\end{array}$ & 7 a 10 \\
\hline & $\begin{array}{l}\text { 2) zones naturelles non protégées (bois, nipisylve, marais } \\
\text { ande) mais classées en zone } \mathrm{N}\end{array}$ & 6 а 9 \\
\hline & $\begin{array}{l}\text { 3) la voirie et les emprises de transport dont l'amènagemen } \\
\text { ntèze le risque d'mondation }\end{array}$ & 5 à 8 \\
\hline & $\begin{array}{l}\text { (4) les aires d'extraction de granulat, en cours d'exploitation } \\
\text { pu abandonnées, dont l'aménagement et la reconversion } \\
\text { grennent compte du risgue d'imondation }\end{array}$ & 5 a 7 \\
\hline \multirow{4}{*}{$\begin{array}{l}\text { III - Espaces anciens } \\
\text { ou récents } \\
\text { n'intégrant pas le } \\
\text { risque d'inondation } \\
\text { et pouvant présenter } \\
\text { une forte } \\
\text { rulnérabilité }\end{array}$} & $\begin{array}{l}\text { 1) espaces d'anciennes politiques urbaines de densificatiog } \\
\text { uu bâti en zone inondable elaborées avant le PPRI (zone } \\
\text { emblayees ou zones toujours a urbaniser : zone AU) }\end{array}$ & 1 a 3 \\
\hline & $\begin{array}{l}\text { 2) espaces en cours d'aménagement urbain conduisant } \\
\text { ggeravation du risque }\end{array}$ & 0 a 2 \\
\hline & $\begin{array}{l}\text { 3) espaces marqués par une occupation illégale (habita } \\
\text { érenne ou temporaire ou toute autre activite anthropique } \\
\text { ype décharge ou stockage de matériaux) }\end{array}$ & 3 a 4 \\
\hline & $\begin{array}{l}\text { 4) secteurs liés aux activites industrielles ou de transpor } \\
\text { lont l'activité se poursuit malgré leur incompatibilité avec la } \\
\text { gestion du risgue d'inondation }\end{array}$ & 1 a 3 \\
\hline
\end{tabular}

16 Cette typologie des espaces créés, recomposés ou ignorés par le risque d'inondation a été croisée avec la grille d'évaluation paysagère de la résilience urbaine précédemment décrite (tableau 2). Il en émane des liens étroits entre classes de résilience et catégories d'espace : les espaces créés par le risque présentent tous une forte résilience (de 12 à 20) signifiant par là que leurs potentialités ne seraient pas compromises par la venue d'un événement hydrologique majeur. De même, les espaces recomposés (de 5 à 10) offrent des possibilités de reconversion importante, même si les dégâts constatés atteindraient des niveaux plus forts. En revanche, les espaces ignorant le risque apparaissent tout à la fois très vulnérables et très peu résilients (de 0 à 4 ). Si l'on ne considère que les Zones d'Aménagement Concerté, comme la ZAC Bel Air à Saint-PryvéSaint-Mesmin lotie dans les années 1980, regroupant des centaines de pavillons de plain-pied dont le haut des toits n'émergerait pas en cas d'une crue de retour 200 ans $^{47}$, on mesure bien le caractère à la fois très récent et préoccupant de l'urbanisation du Val d'Orléans.

\section{Cartographie de la résilience urbaine au risque d'inondation}

Cette démarche croisant typologie des espaces et capacité à faire face au risque permet la confection de trois cartes spatialisant à grande échelle la résilience urbaine au risque d'inondation.

Quasiment entièrement située en zone d'aléas 3 et 4 du PPRI, Saint-Pryvé-SaintMesmin, et la frange inondable d'olivet (au Sud et à l'Est de la fig.1), présentent globalement un système spatial déterminé par l'intensité du risque. À l'Ouest de l'A 71, en zone d'aléa 4, protection de la nature (Réserve naturelle de Saint-Mesmin) et maintien volontaire des espaces agricoles, maraîchers et horticoles pour l'essentiel, 
assurent la résilience de cet espace éminemment vulnérable. À l'est, en zone d'aléa 3, domine une volonté d'optimisation de l'espace constructible (pour reprendre les termes du Projet d'aménagement et de développement durable de Saint-Pryvé-Saint-Mesmin de 2005) marquée tout à la fois par l'apparition d'espaces à forte résilience (espace récréatif et sportif, zone agricole classée en zone N) et par la mise en place de parcs le long du Loiret (futur Parc du Loiret) qui maintiennent les espaces naturels et agricoles en les dotant de voies piétonnières et cyclables. Pourtant, l'ampleur de l'urbanisation à partir des années 1960, puis son accélération dans les années 1990, induisent toujours un très haut niveau de risque et une faible résilience dans les centres-villes des deux communes, comme l'attestent les figurés correspondant au bâti antérieur au PPRI et aux zones d'activités commerciales. Remarquons toutefois, depuis la fin des années 2000, une nette différence de stratégie entre Saint-Pryvé-Saint-Mesmin et olivet. Certes, la première fut longtemps vertueuse en matière de développement durable ${ }^{48}$ : engagée dans de très nombreuses actions en faveur de la revalorisation des milieux d'eau ${ }^{49}$, pionnière dans la sensibilisation au risque de ses administrés ${ }^{50}$, elle fut une commune modèle en matière de résilience spatiale. Mais, depuis les dernières élections municipales, l'équipe en place renoue avec une logique de densification du bâti en zone inondable (même à l'Ouest de l'A 71), anticipant la révision (et le renforcement probable) du PPRI prévue en 2012, sans que pour autant les normes de construction en zone inondable soient très bien déterminées. Olivet, au contraire, qui dispose de très importantes réserves foncières sur le plateau insubmersible et promeut un cadre de vie à forte naturalité le long du Loiret, joue la carte de l'exemplarité en figeant notamment les zones agricoles par leur classement en zone $\mathrm{N}$.

Figure 1 : Résilience urbaine au risque d'inondation. Le cas de Saint-Pryvé-Saint-Mesmin et d'Olivet

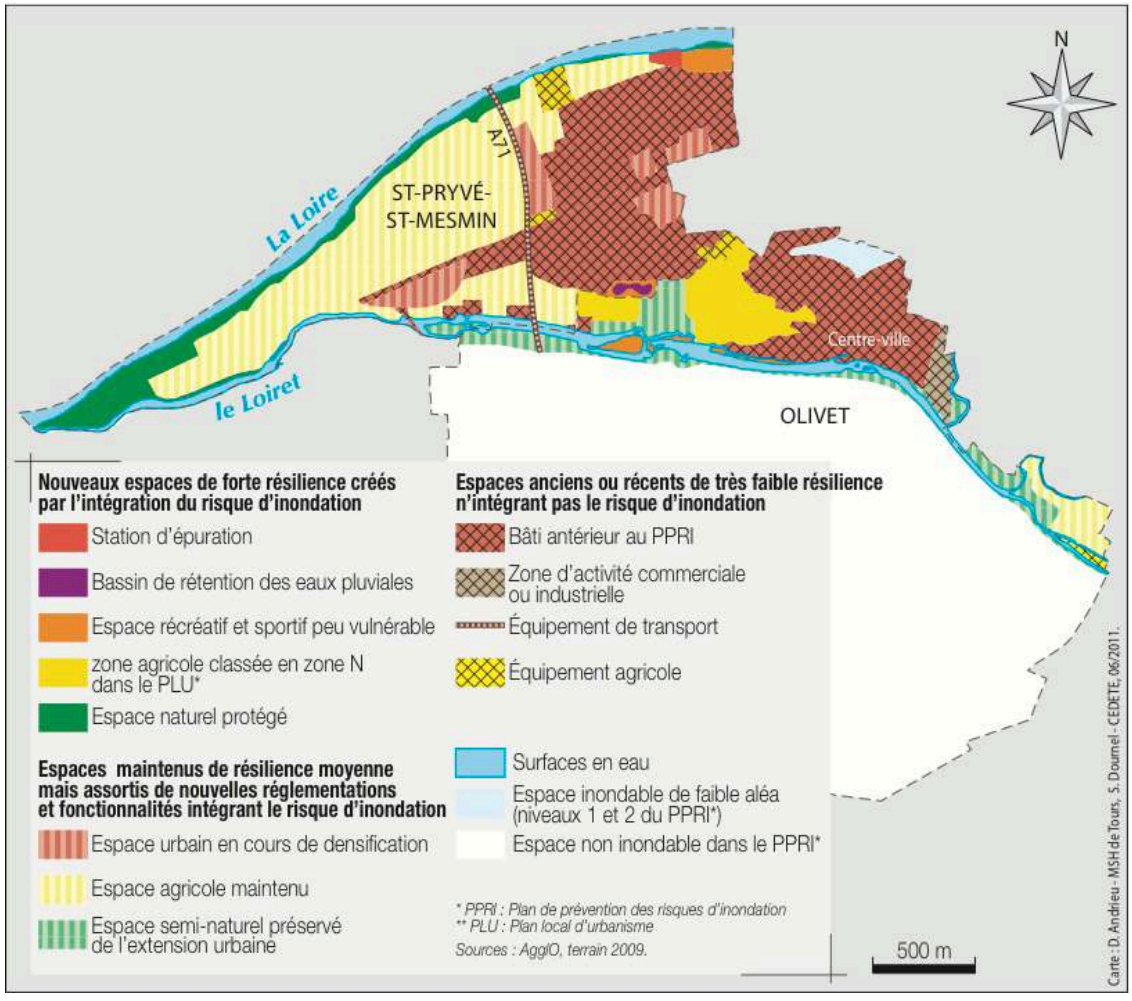

19 En aval de Tours, dans une situation de presqu'île en amont de la confluence entre la Loire et le Cher, la commune de La Riche présente également un fort degré de 
vulnérabilité au risque d'inondation (fig. 2) : la quasi-totalité du territoire communal se trouve en zone inondable, soumis à la forte contrainte imposée par le PPRI ce qui n'a pas empêché une forte progression de la population et une extension du bâti, principalement en secteur d'aléa 1 et $2^{51}$. Dans les zones analysées, d'aléas 3 et 4 , les nouveaux espaces de forte résilience créés par l'intégration du risque sont ainsi très restreints. Il s'agit, en premier lieu de la nouvelle station d'épuration de l'agglomération de Tours, en bord de Cher, dont la restructuration et l'extension ont suivi scrupuleusement les préconisations du règlement du PPRI. La seconde souscatégorie concernée, les zones naturelles, se trouve dans le lit endigué de la Loire qui accueille des écosystèmes identifiés et protégés dans le cadre de Natura 2000. Notons que ce zonage n'est pas spécifique à cette seule commune mais comprend de larges portions de la Loire endiguée. Les espaces maintenus concernent des quartiers en aléa 3 dont la densification est en cours ainsi que des espaces agricoles et semi-naturels dans lesquels les constructions sont limitées ou interdites. La troisième catégorie, qui présente la vulnérabilité la plus forte, est constituée d'habitations dont la construction est antérieure au PPRI et dont l'architecture n'intègre pas le risque d'inondation ainsi que la voie d'accès au périphérique, localisée dans le lit endigué. Il faut préciser qu'il existe un secteur de bâti illégal, également dans le lit endigué (aléa 4), les îles Noires, qui comprend des jardins ouvriers habités temporairement mais également plusieurs habitations occupées tout au long de l'année. Le système spatial s'établit ici parallèlement à l'axe des deux cours d'eau : si le centre est globalement peu résilient (habitat et équipement antérieur au PPRI et densification récente n'intégrant pas totalement le risque), le gradient de résilience augmente quand on s'approche des rives de la Loire ou du Cher. Pourtant, La Riche valorise très faiblement la proximité des deux cours d'eau, la Loire et le Cher, malgré des projets de zones de loisirs inscrits dans son Agenda 21, ou de belvédère touristique dans les secteurs marqués par l'habitat précaire et illégal ${ }^{52}$.

Figure 2 : Résilience urbaine au risque d'inondation. Le cas de La Riche

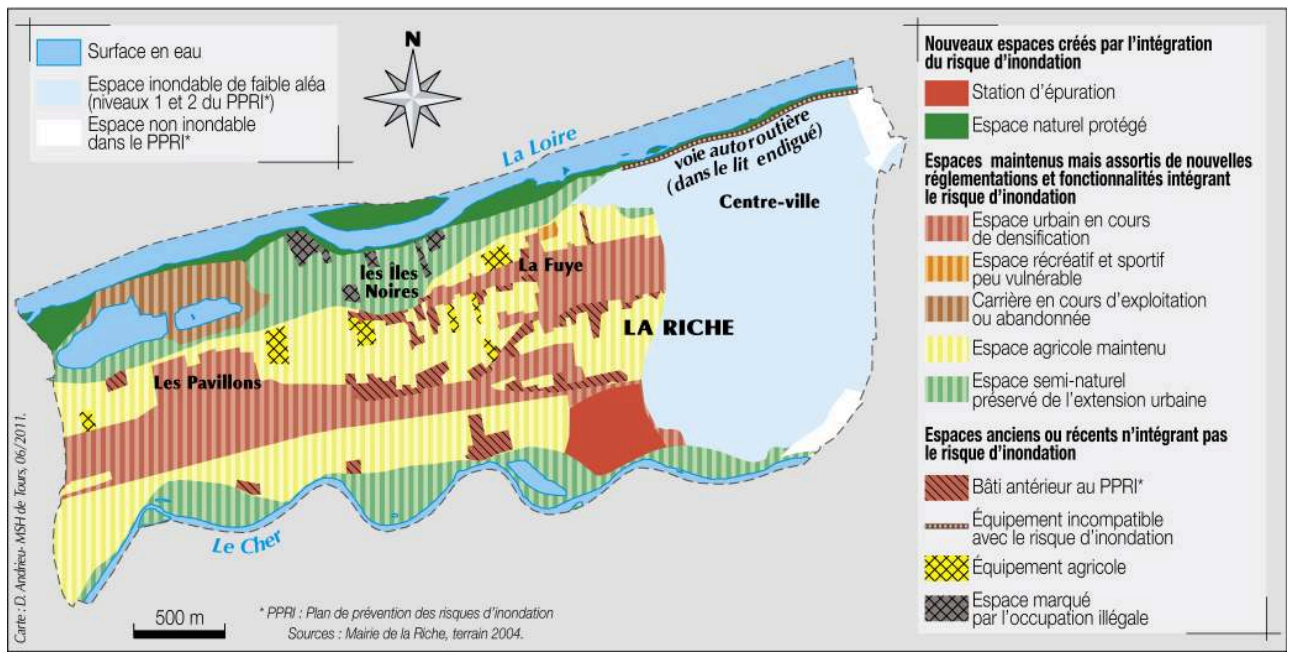

L'agglomération de Blois présente une configuration particulière du fait de la présence d'un déversoir et d'un système de digues protégeant les quartiers inondables de la rive gauche (fig. 3). Le déversoir de la Bouillie, seul du même type localisé en secteur urbain, est un espace de 78 hectares qui doit protéger Blois en cas de crue en écoulant une partie des eaux de la Loire dans le Cosson. S'il a fonctionné régulièrement jusqu'en 
1907, l'absence de crue a entraîné progressivement l'installation de constructions : habitations, jardins familiaux ou cabanons s'y sont accumulés, jusqu'à former un quartier, la Bouillie, en partie de manière illégale, mais aussi avec l'aval d'un permis de construire jusqu'en 1968, date à laquelle le premier plan de surface submersible entre en vigueur. Classé en aléa d'inondation très fort en 1999 dans le PPRI, le quartier de la Bouillie fait alors l'objet d'une opération très originale de désurbanisation ${ }^{53}$ qui a entrainé la mise en œuvre d'une Zone d'Aménagement Différé, longtemps contestée, marquée par la destruction des habitations après leur rachat ${ }^{54}$. Les nouveaux espaces de forte résilience repérés (fig. 3) associent donc de manière quasi-unique protection de la nature (vallées du Cosson et de Loire) et déconstruction volontaire. De même, les espaces maintenus, qui représentent l'essentiel du secteur, conjuguent dans un objectif de ville durable, paysages agricoles péri-urbains, nature ordinaire, équipements culturels ou sportifs et accueil des gens du voyage. Dès lors, l'emprise des faibles résiliences spatiales est-elle limitée à un noyau de bâti antérieur au PPRI qui, dans la ZAD de la Bouillie, devrait d'ailleurs être démoli. Le système spatial urbain fonctionne ici dans une logique de complémentarité entre le quartier de Vienne, que l'on souhaite densifier tout en respectant les normes du PPRI de 1999, voire celles plus strictes du PPRI révisé de 2009, et le quartier de la Bouillie destiné à accueillir des activités et des paysages susceptibles de supporter des crues.

Figure 3 : Résilience urbaine au risque d'inondation. Le cas de Blois

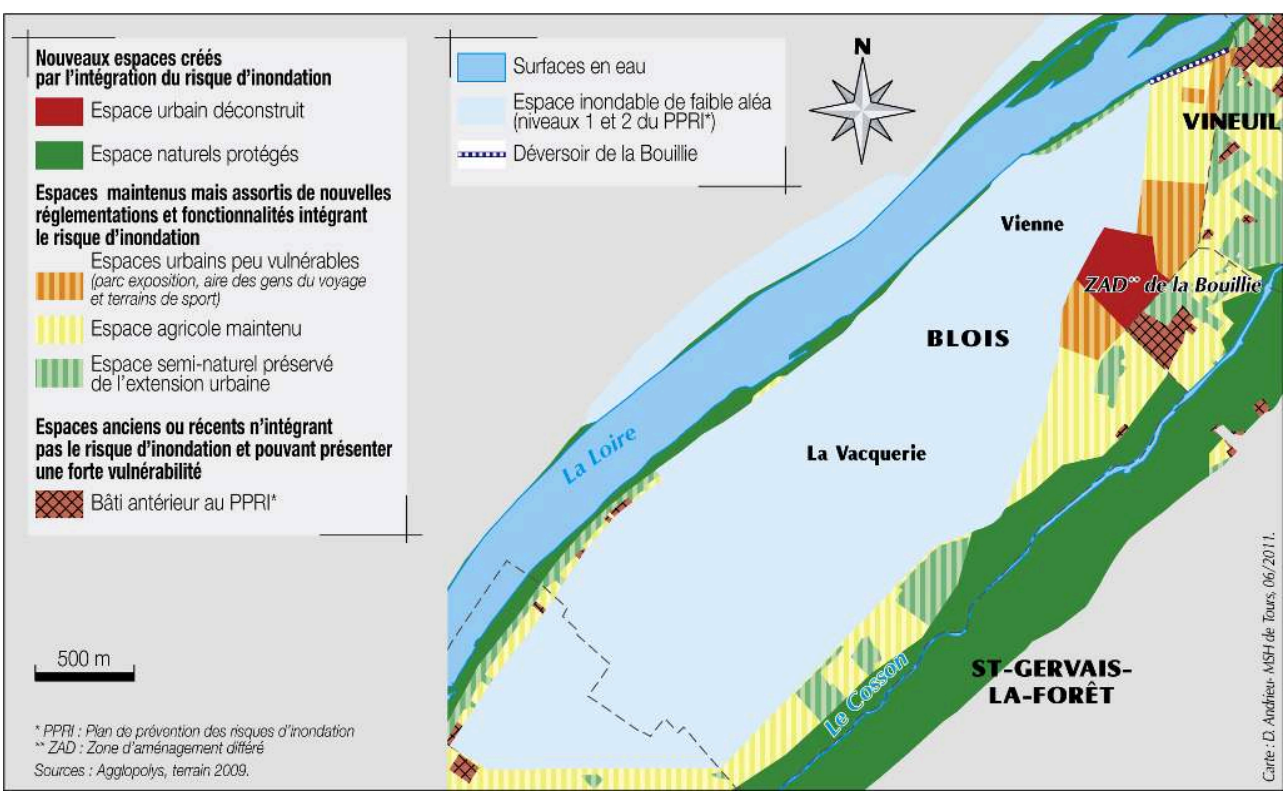

Cette cartographie à grande échelle de la résilience spatiale urbaine fournit ainsi des outils d'aide à la décision en matière d'urbanisme durable et responsable. Construite selon des critères identiques, transposable à l'ensemble des agglomérations ligériennes moyennant de très légères modifications et ajouts, cette méthode autorise également un outil comparatif inédit des politiques urbaines en matière d'intégration du risque d'inondation conduites par les grandes cités s'égrainant le long de la Loire moyenne. Enfin, intégrée dans des SIG, elle peut connaître un prolongement dans l'établissement d'indices établis sur les superficies relatives de chacun des items élémentaires. 


\section{Conclusion}

Motrice de la création de nouveaux paysages et activités, l'intégration du risque d'inondation dans les politiques urbaines des agglomérations ligériennes concourt également à la fabrique de villes plus résilientes. Si cet aspect strictement préventif ne rassemble évidemment pas l'ensemble des objectifs et des pratiques de la notion de ville résiliente, il constitue néanmoins, dès lors que les élus, les services techniques mais aussi les citadins s'emparent de cette réflexion, un puissant facteur de diffusion et de sensibilisation à la constitution de villes durables. Car les liens entre ville résiliente et ville durable sont étroits. La première relève davantage, malgré le succès médiatique du terme résilience, du domaine technique ou scientifique : la complexité même de la notion, mais plus encore celle liée aux solutions et scenarii proposés pour la mettre en œuvre, la confine aux cercles des experts. La seconde, dont se revendique aujourd'hui la grande majorité des grandes cités, partage de fait les mêmes ambitions de développement mais les entoure d'une certaine utopie urbaine plus apte à être entendue du grand public. Quoiqu'il en soit, les deux notions peinent à s'inscrire dans une politique urbaine spatialisée reposant sur des supports cartographiques d'aménagement (SCOT, PLU...). La grille paysagère d'évaluation de la résilience urbaine au risque d'inondation proposée constitue ainsi une avancée vers l'intégration de la résilience, ou du moins de la capacité à faire face au risque, dans les documents d'urbanisme. Outre les réserves formulées en introduction, elle présente toutefois un certain nombre de limites. D'une part, les terrains choisis, entièrement situés en zone inondable de forts aléas, créent un biais naturaliste prononcé. Dès lors qu'il n'est pas envisageable pour des raisons techniques, financières et réglementaires, sauf exception circonscrite à quelques quartiers de prestige, de bâtir des secteurs susceptibles d'être recouverts par 2 à 4 mètres d'eau lors de crues de retour 200 ans, les espaces naturels se voient de facto dotés d'une forte vertu résiliente. Il est donc indispensable d'envisager d'appliquer cette méthode aux espaces affectés par des aléas plus faibles (1 et 2) qui de fait concentrent l'essentiel des hommes et des activités. D'autre part, le choix des marqueurs paysagers de la résilience (au sens de coping capacity) attend sans aucun doute des analyses statistiques multifactorielles plus poussées afin que leurs poids relatifs soient mieux précisés. On retrouve ici toute la difficulté de passer d'un discours qualitatif à une démonstration quantitative. Enfin, cette méthode mérite également d'être appliquée à un plus grand nombre de cités ligériennes afin d'être plus globale mais aussi de mieux appréhender les emboîtements scalaires des systèmes spatiaux. Cette approche constitue donc une étape de la prise en considération des paysages dans la notion de résilience spatiale. 


\section{BIBLIOGRAPHIE}

Aschan-Leygonie C., 1998, Résilience d'un système spatial : l'exemple du Comtat. Une étude comparative de deux périodes de crises au XIXe et au XXe siècles, Thèse de géographie, Université de Paris I

Aschan-Leygonie C., 2000, « Vers une analyse de la résilience des systèmes spatiaux », L'Espace Géographique, 1, pp. 67-77

Berlan-Darque M., Luginbühl Y., Terrasson D., 2007, Paysages : de la connaissance à l'action, Editions QUAE, $316 \mathrm{p}$.

Bravard J.-P., Laurent A.-M., Davallon J., Bethemont J. (éd.), 1995, Les paysages de l'eau aux portes de la ville. Mise en valeur écologique et intégration sociale, Lyon, Programme Rhône-Alpes de Recherche en Sciences Humaines, 331 p.

Burnouf J., Carcaud N., Garcin M., 2003, « La Loire entre mythes et réalités », La Loire revue, no 75, p.12-17

Campanella T. J., 2006, "Urban resilience and the recovery of New Orleans", Journal of the American Planning Association, pp. 141-146.

Cardona O. D., 2004, "The need for rethinking the concepts of vulnerability and risk from holistic perspective: a necessary review and criticism for effective risk management", in Bankoff G. et al., Mapping vulnerability : Disasters, Development and People, Earthscan Publishers, Londres, $212 \mathrm{p}$

Cardona O. D., 2007, Indicators of disaster risk and risk management program for Latin America and the Caribbean summary report updated 2007, Inter-American Development Bank, Sustainable Development Department, Environment Division, Washington, D. C., 54 p.

Chernick H., 2005, Resilient city, Ed. Russel Sage Foundation, 332 p.

Cumming G. S., 2011, Spatial Resilience in Social-Ecological Systems, Hardcover, 243 p.

Cutter S., 2008, A framework for measuring costal hazard resilience in New Jersey Communities, Hazards \& Vulnerability Research Institute, University of South Carolina, Columbia, 12 p.

Cutter S. \& al., 2008, "A place-based model for understanding community resilience to natural disasters", In Global Environmental Change Revue, Local evidence on vulnerabilities and adaptations to global environmental change, Volume 18, Issue 4, pp. 598-606.

Dauphine A., Provitolo D., 2007, « La résilience : un concept pour la gestion des risques », Annales de Géographie, $n^{\circ}$ 654, 2007, pp. 115-125

Dégardin F., 2002, «Urbanisation et inondation : de l'opposition à la réconciliation », Bulletin de l'Association de Géographes Français, no 1, mars 2002, p. 91-103

Dion R., 1961, Histoire des levées de la Loire, édition R. Dion, 312 p.

Dion R., 1978, Le Val de Loire : étude de géographie régionale, Lafitte, 752 p.

Dournel S., 2010,L'eau, miroir de la ville : contribution à l'étude de la requalification urbaine des milieux fluviaux et humides (Bassin parisien, Amiens, Orléans), mémoire de thèse de doctorat en géographieAméhagement-Environnement, Université d'Orléans, dir. B. Sajaloli et G. Giroir, 676 p.

Dournel S., Franchomme M., Sajaloli B., 2011, « Géohistoire d'une résurgence d'eaux troubles : les zones humides urbaines et les cités fluviales de la France du Nord (début XIX $-X^{\mathrm{e}} \mathrm{X}^{\mathrm{e}}$ siècle) », in 
actes du $3^{\text {e }}$ colloque international du GHZH Zones humides et villes d'hier et d'aujourd'hui : des premières cités aux fronts d'eau contemporains, Valenciennes, 25-27/03/2010, Revue du Nord, $\mathrm{n}^{\circ}$ 26, hors série collection histoire, pp. 169-188.

Dournel S., Servain-Courant S., Sajaloli B., Yengue J-L., Andrieu D., 2012 (à paraître), « Le risque d'inondation, moteur de nouveaux paysages urbains? Le cas des villes de Loire moyenne (Orléans, Tours) », in Actes du $135^{{ }^{\mathrm{ème}}}$ Congrès des Sociétés Historiques et Scientifiques «Paysages », Thème 5 Le paysage et l'eau, Neuchâtel, 6-11 avril 2010, éd. du CTHS, sous presses.

Dournel S., Sajaloli B., 2012 (soumis), « Les milieux fluviaux et humides en ville. Du déni à la reconnaissance de paysages urbains historiques », Urban History Review

Dubois-Maury J., Chaline C., 2002, Les risques urbains, Armand Colin, Paris, 208 p.

Folke C., 2006, « Resilience. The emergence of a perspective for social-ecological systems analyses », Global Environmental Change, vol. 16, pp. 253-267

Gralepois M., Servain-CourantS., Sajaloli B., Serrano J., Dournel S., 2011, « Tant va la ville à l'eau. L'intégration du risque d'inondation aux décisions politiques et administratives d'aménagement urbain des agglomérations ligériennes ", actes des $12^{\mathrm{e}}$ rencontres internationales de Liessies, cycle Mémoires et cours d'eau, Hors du lit, aléas, risques et mémoire, Liessies, 23-24 septembre 2010, Revue du Nord, n 16, hors-série collection Art et archéologie, pp. 265-284.

Gunderson L., Holling C. S., 2001, Panarchy: Understanding Transformations in Human and Natural Systems, Island Press, $450 \mathrm{p}$.

Gunderson L., Peterson G., Holling C. S., 2008, "Practicing adaptive management in complex social-ecological systems", pp. 223-245, in Complexity theory for a sustainable future, Edited by J Norberg and G Cumming, Columbia University Press.

Goupil M, 2010, Vulnérabilité des zones bâties inondables et inscriptions paysagères du risque d'inondation dans le val d'Orléans, mémoire de master II GEOGRAM de l'université d'Orléans, dir. B. Sajaloli et S. Servain-Courant, $113 \mathrm{p}$.

Herve D., Laloë F., 2009, Modélisation de l'environnement : entre natures et sociétés, Editions QUAE, 224 p.

Holling C. S., 1973, "Resilience and stability of ecological systems", in Annual Review of Ecology and Systematics, Vol 4, pp.1-23.

Jousseaume V., Mercier D., 2009, « Évaluer la vulnérabilité architecturale de l'habitat en zone inondable : l'exemple du Val nantais », pp. 199-214, in Peltier A. et Becerra S. (éd.), Risques et environnement : recherches interdisciplinaires sur la vulnérabilité des sociétés, Paris, L'Harmattan, $525 \mathrm{p}$.

Kreimer A., Arnold M., Carlin A., 2003, Building safer cities: the future of disaster risk, World Bank Publications, $299 \mathrm{p}$.

Le Bourhis J.P., 2007, « Du savoir cartographique au pouvoir bureaucratique. Les cartes des zones inondables dans la politique des risques (1970-2000) », Genèses, vol. 3, nº 68, pp.75-96

Le Coze J.C., Pettersen K., 2008, Is resilience engineering realist or constructivist?Research and consulting scientist, Accidental Risk Division. National Institute of Industrial Environment and Risks (INERIS), France, $10 \mathrm{p}$.

Lhomme S., Serre D., Diab Y., Laganier R., 2010, « Les réseaux techniques face aux inondations ou comment définir des indicateurs de performance de ces réseaux pour évaluer la résilience urbaine », Bulletin de l'Association de Géographes Français, Géographies (2010), 487-502. 
Meychinet de Richemont N., 2010, « Le risque d'inondation en ville : une impossible territorialisation? » in Bulletin de l'Association des Géographes Français, 2010-4, nº spécial Les grandes métropoles au risque de l'eau : mise en crise et résilience spatiale au Nord et au Sud Moatar F., Ducharne A., Thiery D., Bustillo V., Sauquet E., Vidal J.-P., 2010, « La Loire à l'épreuve du changement climatique », GéoSciences, n²12, décembre 2010, pp. 78-87 [www.brgm.fr]

Murphy L., 2007, "Locating social capital in resilient community-level emergency management", Natural Hazards, Vol. 41, n² 2, pp. 297-315.

Norberg J., Cumming G. S., (eds), 2008, Complexity Theory for a Sustainable Future, Columbia University Press, $315 \mathrm{p}$.

ONU-ISDR, 2010, Pour des villes résilientes, ma ville se prépare, Stratégie Internationale de Prévention des Catastrophes, 24 p. (www.unisdr.org/english/campaigns/campaign2010-2011/documents/ 230_CampaignkitFR.pdf).

Pelling M., 2003, The vulnerability of cities, Ed. Earthscan, 212 p.

Quenault B., Bertrand F., 2010, « Vulnérabilité et résilience aux changements climatiques en milieu urbain », Communication au XXIIIème Colloque de l'Association Internationale de Climatologie Risques et changement climatique, Université de Rennes, 1-4 septembre 2010, Publication acceptée dans les actes du colloque.

Reghezza-Zitt M., 2010, « La résilience dans les politiques françaises de gestion des inondations urbaines ", in Bulletin de l'Association des Géographes Français, 2010-4, nº spécial Les grandes métropoles au risque de l'eau : mise en crise et résilience spatiale au Nord et au Sud

Robert B., 2009, Résilience organisationnelle. Concepts et méthodologies d'évaluation, Presses Internationales Polytechniques, Centre Risque et Performance, Québec, 52 p.

Rode S., 2009, Au risque du fleuve. La territorialisation de la politique de prévention du risque d'inondation en Loire moyenne. Thèse. Paris, Université de Paris Ouest Nanterre La Défense, 481p.

Rode S., Beucher S., 2009, « L'aménagement des territoires face au risque d'inondation : regards croisés sur la Loire moyenne et le Val-de-Marne », Mappemonde, n94.

Rode S., 2010, « Reconquête urbaine de la Loire et risque d'inondation : des représentations aux aménagements urbains ", Géocarrefour, Vol. 85, n 3, p. 221-228.

Salt D., Walker B., 2006, "Resilience thinking: Sustaining Ecosystems and People in a Changing World", Island Presse, $192 \mathrm{p}$.

SAJALOLI B., DOURNEL S., 2008, D'un Orléans, l'autre : étalement urbain et risque d'inondation en Val de Loire, dans Étalement urbain et ville fragmentée à travers le monde, coord. Zaninetti J-M. \& Maret I., Orléans, Presses Universitaires d'Orléans, 2008, vol. 2, Cd-rom, pp. 129-139.

Scarwell H-J, Laganier R., 2004, Risque d'inondation et aménagement durable des territoires, Septentrion Presses Universitaires.

Serrano J., Rode S., Brevet N., Martouzet D., Montembault D., Sajaloli B., Servain-Courant S., 2012 (à paraître), « Les représentations du risque d'inondation par les habitants de la vallée de la Loire, de l'oubli au déni », Colloque Sociétés et catastrophes naturelles, Orléans, 30 septembre-1 octobre 2010, AUTREPART-IRD,

Servain-Courant S., Yengue J.-L., Andrieu D., Rialland C., 2011, « La densification des zones inondables périurbaines, entre opportunité de développement et déni du risque. Le cas de la commune de La Riche dans l'agglomération de Tours (Indre-et-Loire, France) », Revue du Nord, n 26, hors-série collection histoire, pp. 169-188 
Tisseron S., 2009, La résilience, P.U.F., Que sais-je ? , $3^{\text {ème }}$ Ed., Paris, 128 p.

Vale L. J., Campanella T. J., 2005, The resilient city: how modern cities recover from disaster, Oxford University Press, $376 \mathrm{p}$.

Van Der Leew S. E., Aschan-Leygonie C., 2005, A long term perspective on resilience in social-natural systems, in Micro-Meso-macro : Adressing Complex Systems Couplings, World Scientific Publishing, pp. 227-264

Waliser B., Mueller B., Mc Lean C., 2010, La ville résiliente, Groupe de travail de Vancouver, ed. H. Peter Oberlander, $53 \mathrm{p}$.

Verdelli L., Servain-Courant S., Andrieu D., 2006, « Le corridor fluvial ligérien, observatoire privilégié des interactions nature-société, le cas de l'agglomération de Tours », Actes du Colloque international Interaction nature société : Analyses et modèles, La Baule, 3-6 mai 2006, Cd-rom, 2006

Yengue J.-L., Amalric M., Andrieu D., Fournier M., Serrano J., Servain-Courant S., Verdelli L., 2008, Flood risk consideration : a new paradigm. Urban examples in the middle valley of the Loire (France), Flood recovery, innovation and response, edited by D. Porverbs, C.A. Brebbia and E. Penning-Rowsell. Edition WIT Press, 2008.

Yengue J-L., Sajaloli B., Servain-Courant S., Turczi V., 2012 (à paraître), « Architecture et vulnérabilité du bâti en zone inondable dans les communes périurbaines de Loire moyenne, la Riche (37), Saint-Pryvé-Saint-Mesmin et Olivet (45) », actes du Colloque Sociétés et catastrophes naturelles, Orléans, 30 septembre-1 octobre 2010, AUTREPART-IRD.

\section{NOTES}

1. Scarwell et Laganier, 2004

2. Gralepois et al., 2011 ; Servain-Courant et al., 2011

3. Dournel et al., 2011

4. Sajaloli et Dournel, 2008

5. Dournel et Sajaloli, 2012

6. Cumming, 2011

7. Holling, 1973

8. Aschan-Leygonie, 2000 ; Folke, 2006 ; Gunderson et Holling 2011 ; Salt et Walker, 2006 ; Van der Leew, 2005

9. Dauphiné et Provitolo, 2007 ; Tisseron, 2009

10. Campanella, 2006 ; Cutter et al., 2008 ; Murphy, 2007 ; Robert, 2009

11. Chernick, 2005

12. Cutter, 2008; Kreimer et al., 2003 ; Le Coze et Pettersen, 2008 ; Vale et Campanella, 2005 ; Waliser et al., 2010

13. Lhomme et al., 2010 ; Reghezza-Zitt, 2010) ; Quenault et Bertrand, 2010

14. Le Programme Interdisciplinaire de Recherche Ville et Environnement (PIRVE) est un programme de recherche cofinancé par le CNRS et le Ministère de l'Ecologie

15. Dubois-Maury et Chaline, 2002 ; Cardonna, 2004, 2007 ; Pelling, 2003

16. Aschan-Leygonie, 1998

17. Cumming, 2011. Nous ferons notre cette définition américaine de la résilience urbaine

18. Dournel, 2010 ; Dournel et al., 2011

19. www.resalliance.org/ cf : Gunderson et Holling, 2001 
20. Les quatre phases sont : croissance, phase $\mathrm{r}$; conservation, phase $\mathrm{K}$; déstabilisation, phase $\Omega$; réorganisation, phase $\alpha$.

21. Gunderson et al., 2008 ; Norberg et Cumming, 2008

22. Berlan-Darque et al., 2007

23. Programme Évaluation de la Vulnérabilité du Bâti et de la Biodiversité financé par le Plan Loire (FEDER- Établissement Public Loire ; programme VHILZIVAL consacré à la vulnérabilité sociale en zones inondables financé par les universités d'Orléans et de Tours; programme PATRA Patrimoine et trajectoires paysagères des vallées ligériennes financé par le Plan Loire et soutenu par la Zone Atelier Loire

24. Servain-Courant et al., 2011 ; Yengué et al., 2012 ; Dournel et al., 2012

25. Rode et Beucher, 2009 ; Serrano et al., 2012 ; Yengué et al., 2008

26. Sajaloli et Dournel, 2008

27. Gralepois et al., 2011

28. Dion, 1961, 1978 ; Sajaloli et Dournel, 2008

29. Yengué et al., 2008

30. www.floodresiliencity.eu

31. Cumming, 2011

32. Nous reprenons ici littéralement l'expression de G.S. Cumming (2011) p. 49 : «Larger-scale processes should ultimately be more predictibable than fine-scale processes, because the average over finescale variance (which is often a form of "noise ») and provide a more reliable indicator of mass trends "

33. Meychinet de Richemond, 2010

34. Le Bourhis, 2007

35. Rode, 2009 ; Verdelli et al., 2006 ; Jousseaume et Mercier, 2009

36. Bravard et al., 1995 ; Dégardin, 2002

37. Projet de recherche Evaluation de la Vulnérabilité du Bâti et de la Biodiversité financé par le Plan Loire (FEDER- Etablissement Public Loire)

38. Goupil, 2010

39. Rode, 2010 ; Serrano et al., 2011

40. www.r.inondation-loire.fr, site sur le risque d'inondation de la Loire appartenant à l'Établissement Public Loire, est à destination des particuliers et des professionnels. www.prim.net est le site national officiel de la prévention des risques majeurs, créé à l'initiative de la Direction générale de la prévention des risques

41. Études réalisées par la plate forme «Prévention des inondations » du Plan Loire, http:// www.plan-loire.fr/fr/les-plates-formes/prevention-des-inondation

42. Burnouf et al., 2003

43. Sajaloli et Dournel, 2008

44. Gralepois et al., 2011

45. Dournel et al., 2012

46. Gralepois et al., 2011

47. Yengué et al., 2012

48. Gralepois et al., 2011

49. Ceci renvoie à l'extension de la Réserve naturelle de Saint-Mesmin et à la recréation de la continuité des cheminements le long du Loiret

50. Commune-test pour l'Établissement Public Loire avec la mise en place du logiciel de gestion de crises OSIRIS-inondation qui est destiné à aider les communes et les gestionnaires dans l'élaboration du Plan Communal de Sauvegarde, volet Inondation, Saint-Pryvé-Saint-Mesmin constitua également un réseau de correspondants en cas d'alerte

51. Servain-Courant et al., 2011

52. Rode et Beucher, 2009

53. Yengué et al., 2008 


\section{RÉSUMÉS}

La prise en compte du risque d'inondation dans les politiques urbaines des trois principales villes de Loire moyenne (Orléans, Blois, Tours) et les modalités paysagères de cette inscription sont approchées par l'élaboration d'une grille d'analyse paysagère commune permettant l'étude comparative de l'intégration du risque dans les trois agglomérations étudiées. À partir des documents cartographiques ou iconographiques disponibles et d'enquêtes de terrain, les aménagements urbains récents ou anciens situés en zone inondable de forts aléas (niveaux 3 et 4 du Plan de prévention du risque d'inondation, PPRI) ont été caractérisés. Dès lors, il est possible de voir, d'une part, en quoi les politiques urbaines intégrant le risque d'inondation sont susceptibles de façonner de nouveaux paysages urbains et de nouvelles pratiques de la ville et, d'autre part, d'évaluer les caractéristiques des aménagements toujours vulnérables ou aggravant les risques d'inondation. Cette analyse débouche sur une typologie des espaces créés, recomposés ou ignorés par l'intégration du risque dans les politiques urbaines depuis le lancement des PPRI au début des années 2000. À partir de cette typologie, une grille paysagère d'évaluation de la résilience urbaine au risque d'inondation a été confectionnée grâce à des marqueurs révélant la vulnérabilité ou au contraire l'adaptation de l'unité paysagère à l'aléa encouru. Chaque marqueur est doté d'une appréciation chiffrée permettant les comparaisons inter-sites et attribuant à chaque catégorie de paysage un indice de résilience. Les synthèses spatiales sont enfin réalisées grâce à l'utilisation de trois Systèmes d'Informations Géographiques homogènes pour chacun des sites retenus : il en ressort une première appréciation à la fois spatialisée et hiérarchisée de la résilience urbaine au risque d'inondation.

The integration of the risk of flooding in the urban policies, which invoke besides sustainable development, contributes to move the perception of local authorities, and the big private actors, on the not yet built flood-risk areas. It results together of this new urban stakes (nature in city, role of natural corridor, social diversity...) and also new functional areas with the morphological characteristics unpublished (spaces of the seasonal leisure, wildlife sanctuary in the city, agrarian and market garden zones in the cities and on their outskirts). By integrating economic, social and environmental functions of the wetlands, this paper seeks at thirst to draw up a typology of the landscaping mutations noticed in the flooded places of many urban areas of the Loire Valley (Orléans, Blois, Tours) since the instauration of the Plans of Prevention of the Risk of Flooding at the beginning of 2000's. This paper tries also to elaborate a landscaped scale to value the urban resilience at the risk of the flooding. This work spring from a project on the evaluation of the vulnerability of the building in the flooded places, in the Loire Valley to Nevers to Nantes (Plan Loire, European Regional Development Fund), which is widening the scope of the un-built places.

We first treat the consideration of the risk of flooding in the urban policies and in the landscaping effects of its inscription by the elaboration of a same landscaped scale to value, in a comparative study, the integration of the risk in the studied cities. We defined and referred in the Geographic Information System the urban and flooded places recently designed (urban parks, green spaces) or formerly designed (agrarian and market zones, activities zones), from cartographic and iconographic documents available for consultation and from local 
investigations. From then on, we can see how the urban policies, which integrate the risk of flooding can make new urban landscapes and new urban practices. In the same way, we can value the characteristics of urban places which always are vulnerable or which aggravate the risk of flooding. Our research result a typology of urban places created changed or ignored for the integration of the risk in the urban policies since the instauration of the Plans of Prevention of the Risk of Flooding.

This typology uses elements revealing the vulnerability or on the contrary the adaptation of the landscaped unity, which leads to the construction of a landscaped scale to value the urban resilience at the risk of the flooding. Every element is characterised by a numbered appreciation, which allows comparisons between the studied urban places and attributes to every category of landscape an index of resilience. A synthesis, basing on the use of four homogeneous Geographic Information Systems, is at last realising for every studied cities of the Loire Valley: the result of the investigation shows at the same time a territorialised, hierarchical and comparative of the urban resilience at the risk of flooding of this studied cities.

\section{INDEX}

Mots-clés : agglomérations ligériennes, indice paysager de résilience urbaine, résilience spatiale, risque d'inondation, ville durable

Keywords : Loire' cities, risk of the flooding, spatial indication of urban resilience, spatial resilience, sustainable city

\section{AUTEURS}

\section{BERTRAND SAJALOLI}

Maître de conférences en géographie, Université d'Orléans, UFR Lettres, Langues et Sciences Humaines, CEDETE (EA 1210), 10 rue de Tours, BP 46527, 45065 Orléans cedex 2bertrand.sajaloli@univ-orleans.fr

\section{SYLVIE SERVAIN-COURANT}

Maître de conférences en géographie, École Nationale Supérieure de la Nature et du Paysage de Blois, UMR6173 CITERES (CNRS et Université de Tours), servain@ensnp.fr

\section{SYLVAIN DOURNEL}

Post-doctorant, département de géographie, Université d'Orléans, CEDETE (EA 1210)

sylvain.dournel@univ-orleans.fr

\section{DOMINIQUE ANDRIEU}

Ingénieur de recherche, UMS1835 Maison des Sciences de la Ville, de l'Urbanisme et des Paysages (CNRS et Université de Tours), dominique.andrieu@univ-tours.fr 